\title{
Position Statement for Mental Health Care in Long-Term Care During COVID-19
}

Claire Checkland, BA ${ }^{\mathrm{a}}$, Sophiya Benjamin, MBBS ${ }^{\mathrm{b}}$, Marie-Andrée Bruneau, MD MSc ${ }^{\mathrm{c}}$, Antonia Cappella, $\mathrm{MD}^{\mathrm{d}}$, Beverley Cassidy, $\mathrm{MD}^{\mathrm{e}}$, David Conn, $\mathrm{MB} \mathrm{ChB}^{\mathrm{f}}$, Cindy Grief, $\mathrm{MD}, \mathrm{MSc}^{\mathrm{f}}$, Alvin Keng, MD $^{\mathrm{g}}$, Julia Kirkham, MD, MSc ${ }^{\mathrm{h}}$, Popuri Krishna, MBBs ${ }^{\mathrm{i}}$, Lisa McMurray, MD ${ }^{\mathrm{j}}$, Kiran Rabheru, $\mathrm{MD}^{\mathrm{j}}$, Marie-France Tourigny-Rivard, $\mathrm{MD}^{\mathrm{j}}$, Dallas P. Seitz, MD, $\mathrm{PhD}^{\mathrm{h}}$

${ }^{a}$ Canadian Coalition for Seniors Mental Health, Toronto, $\mathrm{ON} ;{ }^{b}$ Department of Psychiatry and Behavioural Neurosciences DeGroote School of Medicine, McMaster University,Hamilton, ON; ${ }^{c}$ Geriatric Psychiatry division, Department of Psychiatry, University of Montreal, Montreal, QC; ${ }^{d}$ Department of Psychiatry, University of Alberta, Edmonton, AB; ${ }^{e}$ Seniors Mental Health, Department of Psychiatry, Dalhousie University, Halifax, NS; ${ }^{f}$ Baycrest Health Sciences and University of Toronto, Toronto, ON; ${ }^{g}$ Geriatric Psychiatry Residency Program, University of Toronto, Toronto, ON; ${ }^{h}$ Department of Psychiatry, Cumming School of Medicine, University of Calgary, Calgary, AB; ${ }^{i}$ Department of Psychiatry, Northern Ontario School of Medicine, Sudbury, ON; ${ }^{j}$ Department of Psychiatry, University of Ottawa, Ottawa, ON, Canada

https://doi.org/10.5770/cgj.24.514

\begin{abstract}
COVID-19 has disproportionately impacted older adults in long-term care (LTC) facilities in Canada. There are opportunities to learn from this crisis and to improve systems of care in order to ensure that older adults in LTC enjoy their right to the highest attainable standard of health. Measures are needed to ensure the mental health of older adults in LTC during COVID-19. The Canadian Academy of Geriatric Psychiatry (CAGP) and Canadian Coalition for Seniors' Mental Health (CCSMH) have developed the following position statements to address the mental health needs of older adults in LTC facilities, their family members, and LTC staff. We outlined eight key considerations related to mental health care in LTC during COVID-19 to optimize the mental health of this vulnerable population during the pandemic.
\end{abstract}

Key words: COVID-19, coronavirus, nursing home, longterm care, mental health, dementia

\section{KEY POINTS:}

1. Mental health care is an essential medical service that must be maintained during any pandemic.

2. Older adults living in LTC facilities have the right to mental health, medical care, and social services, regardless of their age, the presence of dementia, or a diagnosis of other mental health problems and illnesses.

3. LTC facilities must be provided with the technology and resources necessary for provision of essential virtual medical services, and for the maintenance of family and social connections when in-person visits are restricted.
4. Infection control measures intended to reduce the spread of infectious diseases must also balance the impact of these measures on the quality of life and dignity of LTC residents.

5. Supporting the mental health of LTC staff is critical to effectively managing pandemics in LTC.

\section{INTRODUCTION}

Throughout the pandemic, Canada has consistently had one of the highest global proportions of all COVID-19 deaths occurring among LTC residents. ${ }^{(1-3)}$ While many recommendations related to improving care for LTC residents during COVID-19 have been created both nationally ${ }^{(4-8)}$ and internationally, ${ }^{(9,10)}$ none have focused on the mental health implications associated with COVID-19 in LTC. Mental disorders including dementia, depression, and anxiety are common in LTC. ${ }^{(11,12)}$ Despite this high need for mental health supports among LTC residents, their access to mental health care was poor even prior to the pandemic. ${ }^{(13,14)}$

Several factors have contributed to a potential worsening of the mental health of LTC residents during COVID-19. Ageism, ${ }^{(15)}$ predating COVID-19, is contributing to the adverse mental health impacts related to COVID-19 in LTC in Canada. $(16,17)$ The COVID-19 pandemic has identified long-standing consequences of ageism in LTC due in part to measures implemented to manage COVID-19 in this setting. ${ }^{(9,18)}$ Furthermore, chronic staff shortages, limited staff education about dementia and mental health, and poor access to psychiatric services in LTC have all contributed to the current LTC crisis. ${ }^{(19)}$ While these serious problems have received media attention, ${ }^{(20)}$ these 
concerns have not been reflected adequately in recommendations meant to address the impact of COVID-19 in LTC. Already concerns have arisen that quality of mental health care in LTC facilities has deteriorated further. ${ }^{(21)}$

This position paper highlights the mental health care needs of older persons living in LTC settings during COVID-19 and can be applied to other serious outbreaks.

\section{METHODS}

The Canadian Academy of Geriatric Psychiatry (CAGP) and Canadian Coalition for Seniors Mental Health (CCMSH) established a working group to develop position statements on mental health care for older adults in LTC settings during COVID-19. A call for potential working groups' participants was distributed to CAGP members. A final working group consisting of 14 individuals representing geriatric psychiatrists and trainees from different provinces, care settings, and career stages was established. This working group initially met in July, 2020 to identify the scope and anticipated outputs for this project, and met three additional times during the development of the position statements.

The working group reviewed existing recommendations related to care of older adults during COVID-19 including documents related to LTC. The working group developed preliminary position statements and reviewed evidence related to each statement. The electronic database MEDLINE was searched from 2020 onwards to identify relevant articles. This was supplemented by manual searches of journals which have published extensively on the topics of COVID-19. Google Scholar and Google were also used to identify additional relevant documents including media articles. The final position statements and accompanying evidence were arrived at by consensus by the working group.

\section{POSITION STATEMENTS}

\section{Statement 1: Mental health care in LTC settings is an essential service.}

While access to medical services may need to be adapted during COVID-19 and other outbreaks in keeping with public health requirements, mental health care in LTC is an essential service. During the initial outbreak of COVID-19 in Canada, preparations for an anticipated influx of COVID-19 patients into acute care led to the cancellation of many medical and surgical services deemed non-essential at the time. In addition, many hospital-based outpatient programs and procedures, such as ECT, were suspended or reduced to prevent spread of COVID-19 virus. ${ }^{(22)}$ As a result, many individuals with mental health conditions in LTC settings had mental health assessments or treatments delayed or cancelled during the first six months of the COVID-19 pandemic in Canada.

While mental health assessments or treatments can be delayed for some individuals due to infection control practices, many individuals with mental health problems and illnesses in LTC facilities require urgent access to mental health services. These services may include outpatient visits, outreach services, inpatient admission, and the ability to initiate or continue hospital-based mental health treatments such as ECT. ${ }^{(23)}$ COVID-19 has disrupted mental health services in most countries. ${ }^{(24)}$ While COVID-19-related restrictions to health-care provider visits have disrupted mental health services in most LTC facilities, the impact of COVID-19 on access to mental health services in LTC is unknown. Therefore, health systems need to provide the necessary safeguards and processes to allow ongoing mental health care for older adults in LTC while at the same time protecting LTC residents from the risks associated with COVID-19 infection.

\section{Statement 2: Factors such as age, the presence of dementia, or a diagnosis of mental health problems or illnesses are not reasons to exclude individuals from having access to resources such as emergency departments or inpatient hospitalization.}

The COVID-19 pandemic has challenged health-care organizations to prepare for increased demands on acute care. Crisis triage protocols have been developed, but often use survivability to determine allocation of health care resources. (25) This approach may stigmatize older adults, and those with chronic illness or disability. (26) Older adults who contract COVID-19 have higher mortality rates, especially in the presence of chronic medical conditions. ${ }^{(26)}$ LTC residents have a high burden of such illnesses, and the presence of frailty and dementia further compounds their vulnerability. $(27,28)$

On admission to LTC, advanced care planning and goals of care discussions should occur between individuals, substitute decision makers (SDMs), and health-care teams. Furthermore, these discussions should be routinely revisited. ${ }^{(29)}$ Individuals and SDMs should be provided with the information necessary to make informed choices about remaining in LTC or being transferred to acute care, and about the level of care that is to be received. Individuals and their families must be supported through this process and able to express their wishes, while also being made aware of the significantly lowered survival rates among older adults with multimorbidity who become infected with COVID-19. The presence or absence of cognitive impairment should not in itself preclude access to treatment, but should factor into decisions about goals of care. It is essential that stigma and ageism do not influence these decisions.

\section{Statement 3: Restrictions on in-person visits to LTC must include measures that address the potential negative effects of these restrictions on the mental health, quality of life, and dignity of LTC residents and their families.}

Restrictions to LTC visits may need to be implemented rapidly by LTC facilities and public health organizations to minimize the spread of COVID-19 in communities or LTC where risk of transmission is high. While some restrictions on LTC visits will be required during infectious disease outbreaks, the least restrictive approaches to visitation permitted during pandemics should be made available to all LTC residents. 
Restrictions intended to prevent the spread of COVID-19 have significant effects on the social network of individuals in LTC, and plans to minimize potential negative impacts of social isolation need to be considered in conjunction with any restrictions to visits in LTC.

Plans to minimize the negative impacts of isolation on these individuals and their family should be in place, including adherence to compassionate practices for ensuring social connection and care. ${ }^{(30)}$ Most effective non-pharmacological interventions for dementia in LTC facilities, including oneto-one visits provided by family members or other individuals, require two to three interactions per week for sustained benefits on mood and behaviours to be observed between interactions. ${ }^{(31)}$ While facility-level restrictions on visits may not permit this frequency of visits during COVID-19 or other outbreaks, LTC facilities should offer a minimum of at least weekly contact with a family member or designated support persons through virtual means or a safe in-person visit with appropriate personal protective equipment. Ideally, patients would be able to receive visits in accordance with their needs and not be limited to an arbitrary number.

Specific plans for all infectious outbreaks in LTC need to include not only infection prevention measures, but also a plan to maintain nursing care, social contact, and activities that support the mental health of the residents and prevention of unnecessary physical and cognitive decline. Families and caregivers of LTC residents should be involved in the development of these plans. While these measures are often implemented during infectious disease outbreak in LTC facilities, during COVID-19 these restrictions occurred for prolonged periods of time and had significant impacts for the residents of LTC. These included loneliness, anxiety, feelings of insecurity, and suicidal thoughts, as well as physical and cognitive decline. ${ }^{(13)}$

Caregivers have also experienced significant stress when they could no longer be physically present to support their family members in LTC facilities. This was compounded in many situations by a lack of information and communication from LTC facilities about their loved ones. LTC facilities should implement processes to provide LTC residents, their families and caregivers, and staff with timely information related to the COVID-19 pandemic. LTC facilities may consider providing access to electronic charts or secure communications for family members. All LTC facilities should have LTC staff who are key contacts and can provide timely information to LTC residents, their families, and caregivers.

\section{Statement 4: Appropriate communication technology and human resources must be available to allow communication between LTC residents and individuals located outside a resident's LTC facility.}

Access to virtual communication technology (e.g., phone, tablet, or computer-based videoconferencing) has been identified as critical to ensuring that LTC residents are able to communicate with their families, friends, and health service providers who are unable to visit in-person at a LTC. ${ }^{(32,33)}$ Utilization of telemedicine (services delivered by physicians) and telehealth services (delivered by other health-care providers) have been identified as key to maintaining access to health care during COVID-19(34) and reducing disease transmission. ${ }^{(35)}$ Older adults in LTC facilities may suffer the effects of "double burden of exclusion" by having restrictions to in-person visits and limited access to technology needed for other methods of communication. ${ }^{(36)}$ Unfortunately, many LTC facilities have limited access to the technology, expertise, and human resources necessary to support this form of communication at all or as frequently as would be optimal for an individual's needs. ${ }^{(33)}$

Each LTC facility should have the infrastructure to support virtual visits with family and health-care professionals. At a minimum, this should include high-speed internet connection, and the provision of portable devices (e.g., tablets or laptops) for LTC residents who do not have access to their own personal device. All LTC staff should have basic knowledge of facilitating virtual visits using commonly used platforms. The unique needs of older adults with dementia, mental illnesses, and comorbid sensory impairments must be accommodated in these visits. Each LTC facility should have two or more staff per resident care unit trained in facilitating secure and private virtual health-care visits that follow required privacy regulations. Interprofessional teams based outside of LTC facilities should also receive training in conducting virtual assessments, and be provided with the technology necessary to complete assessments.

\section{Statement 5: Processes to allow exceptions to restrictions on caregiver visits must be available to individuals who may experience severe harm to their mental health and quality of life when visits are restricted in LTC.}

Facility-level policies restricting access to LTC visits may be required periodically during COVID-19 and the least restrictive approaches should be implemented whenever possible. However, even when least restrictive approaches are implemented, there may be specific individuals in LTC who will have a disproportionate deterioration in their mental health or quality of life because of these facility-level policies. As new policies for the integration of family caregivers at LTC facilities are developed, they should follow a person-centred approach. Processes must be in place for exceptional circumstances where family caregivers are required as part of an individual's mental health-care plan. Families, caregivers, geriatric psychiatrists, and LTC mental health service providers should be consulted in the development of these policies and be included in the review of such exceptional circumstances.

LTC facilities did not allow any visitors in the early stages of the COVID-19 pandemic, which resulted in an overwhelming response from LTC residents, their families, and caregivers about the negative impact of these restrictions. ${ }^{(37)}$ Early studies examining the reintroduction of visitors in LTC through carefully implemented policies have demonstrated success, and show that these visits have not led to a significant increase in the rates of COVID-19 infection. ${ }^{(38)}$ There are emerging recommendations on the reintegration of family 
caregivers into LTC, which require rapid dissemination to adopt evidence-based visitation policies that provide a better balance of infection control measures with quality of life. ${ }^{(39)}$

Failure to utilize family presence in LTC facilities to support older adults exacerbates the current gaps in the LTC workforce. It is estimated that approximately 750,000 Canadians provide care to a family member in a LTC setting, with over $20 \%$ of them providing over $10 \mathrm{hrs}$ per week of care including personal care. ${ }^{(40)}$ LTC residents have discussed their experiences over the last six months, with some describing their experience as "devastating, emotional, terror awakened, muzzled, trapped, broken-spirited, and boredom". ${ }^{(37)}$ Research into the impact of these restrictions in LTC facilities should include direct interviews with residents and family caregivers. ${ }^{(37)}$

\section{Statement 6: All LTC facilities must have adequate training, staffing, and resources to assess and treat common mental health conditions during COVID-19 and other infectious disease outbreaks.}

While mental health conditions are common in LTC settings, ${ }^{(11)}$ access to mental health and psychiatric services in LTC is limited. ${ }^{(14)}$ Canada has a shortage of geriatric psychiatrists which is unlikely to improve substantially in coming years. ${ }^{(41)}$ Research has suggested that COVID-19 has resulted in an increase in behavioural symptoms of dementia in LTC resulting in an even greater need for mental health care. ${ }^{(42,43)}$ It is therefore important that all LTC facilities have adequately trained staff and sufficient resources to assess and treat common mental health conditions during COVID-19 and similar periods of infectious disease outbreaks when access to mental health resources is even more limited.

Staff training approaches are among the best supported interventions for addressing behavioural symptoms of dementia in LTC settings. ${ }^{(31)}$ Guidelines recommend that all LTC facilities provide staff with training in the assessment and management of common mental health disorders ${ }^{(44,45)}$ such as delirium and depression, ${ }^{(44)}$ management of neuropsychiatric symptoms, and responding to emergent mental health crises. ${ }^{(46)}$ These training programs require access to trainers, as well as resources, and the permission for staff to attend these courses as part of their paid employment, while ensuring an adequate number of staff are available to support the ongoing needs of LTC residents. Some LTC homes may have mental health champions or embedded mental health resources provided by either the LTC facility or through partnerships with provincial mental health programs. Mental health care provided by LTC staff can be supplemented by regional outreach programs or telemedicine. ${ }^{(47)}$

\section{Statement 7: Governments, LTC, and mental health service providers must ensure that staff working in LTC during the COVID-19 pandemic have adequate access to mental health supports and programs to support staff wellness.}

Staff working in LTC settings were experiencing significant stress and devaluation due to chronic understaffing of LTC facilities prior to COVID-19. COVID-19 has contributed additional stressors related to a worsening of staffing shortages, initial shortages of PPE, and ongoing risks of staff contracting COVID-19, and the high death rates in COVID-19-affected facilities. ${ }^{(48,49)}$ Each individual may respond to the new stressors associated with COVID-19 differently and many experience mental health consequences. ${ }^{(50)}$

To mitigate these negative sequelae, LTC facilities must promote and maintain the mental health and well-being of frontline staff by ensuring measures are taken to adequately support staff, ensure their safety, and recognize the value of their work. ${ }^{(49)}$ This includes access to appropriate personal protective equipment and educational programs to ensure its proper use. ${ }^{(5)}$ Psychoeducation on caregiver burnout, stress management, and mental health disorders should be incorporated in LTC job training and regularly reviewed to promote staff resilience. Sick leave and employee assistance programs should be made available to staff working in LTC facilities impacted by COVID-19. Clear guidance from LTC facility leadership, optimization of human resource planning, and adoption of clinical practices to minimize the impact of COVID-19 in LTC are also important in supporting LTC staff. ${ }^{(49)}$

\section{Statement 8: Measures of mental health and quality of life in LTC facilities must be systematically evaluated during COVID-19, and strategies implemented to understand and remediate adverse mental health outcomes when they are identified.}

Mental health and quality of life must be systematically evaluated to assess the effects of the COVID-19 pandemic on LTC residents. This monitoring will help identify early signs of worsening mental health symptoms within individual LTC residents and facilities, and identify individuals and facilities that may require additional supports or resources, similar to how LTC with COVID-19 outbreaks are currently triaged for additional supports based on their needs.

While mortality and infection rates may reflect the most important measures for monitoring of the impact of COVID-19 in other settings, they capture only part of the effects of COVID-19 in LTC. One of the most dramatic effects of COVID-19 in LTCs has been the increase in social isolation of residents due to restrictions on visitors and residents' ability to leave their care home. Social isolation has adverse mental health outcomes such as worsening of depression, cognitive decline, and increasing behavioural symptoms of dementia. ${ }^{(51)}$

Measurement is the basis of monitoring quality of care and overall health status, and identifies opportunities for improving quality of care amidst the COVID-19 pandemic. Monitoring quality of life, functioning, and mental health can often be done without creating new or onerous data collection. For example, the Minimum Dataset Resident Assessment Instrument (MDS-RAI) is a routine measure used in LTC, which includes quality indicators measuring depression, ${ }^{(52)}$ behavioural symptoms, ${ }^{(53)}$ and cognition. ${ }^{(54)}$ Incorporating these and other relevant measures of LTC resident health status as part of COVID-19-related outcomes would provide a more accurate picture of the effects of COVID-19 in LTC. 


\section{CONCLUSION}

All Canadians have been affected by the COVID-19 pandemic and none more so than LTC residents. As the COVID-19 pandemic continues, we anticipate that the mental health of LTC residents, their families, and LTC staff will continue to be significantly impacted. Our position statements provide actionable strategies and priorities to minimize the impact of COVID-19 on the mental health of individuals in LTC. Collectively, we can learn from our actions in the past and make plans for a future that better meets the mental needs of individuals in LTC during COVID-19 and similar disease outbreaks.

\section{ACKNOWLEDGEMENTS}

This position statement was partially funded through the Canadian Consortium on Neurodegeneration in Aging funded by the Canadian Institutes of Health Research

\section{CONFLICT OF INTEREST DISCLOSURES}

The authors declare that no conflicts of interest exist.

\section{REFERENCES}

1. Government of Canada. Coronavirus 2019 (COVID-19) daily epidemiology update. Ottawa, ON: Government of Canada. Accessed September 28, 2020.

2. Barnett ML, Grabowski DC. Nursing homes are ground zero for COVID-19 pandemic. JAMA Health Forum. 2020;1(3):e200369

3. Hsu AT, Lane N. Impact of COVID-19 on residents of Canada's long-term care homes-ongoing challenges and policy response. London, UK: Int Long Term Care Policy Netwk; 2020. Available at: https://tcCOVID.org/2020/04/15/impact-of-COVID-19-onresidents-of-canadas-long-term-care-homes-ongoing-challengesand-policy-response/

4. Healthcare Excellence Canada. Re-integration of family caregivers as essential partners in care in a time of COVID-19. Ottawa, ON: Healthcare Excellence Canada; 2020. Available at: https://www.cfhi-fcass.ca/about/news-and-stories/news-detail/2020/07/08/re-integration-of-family-caregivers-as-essentialpartners-in-care-in-a-time-of-COVID-19

5. Canadian Centre for Policy Alternatives. Re-imaging long-term residential care in the COVID-19 crisis. Ottawa, ON: the Centre; 2020. Available from: https://www.policyalternatives.ca/publications/reports/re-imagining-long-term-residential-care-COVID19-crisis

6. Canadian Institute for Health Information. Pandemic experience in the long-term care sector: How does Canada compare with other countries? Ottawa, ON: Canadian Institute for Health Information; 2020. Available from: https://www.cihi.ca/sites/ default/files/document/COVID-19-rapid-response-long-termcare-snapshot-en.pdf

7. Government of Canada. Interim guidance: care of residents in long term care homes during the COVID-19 pandemic. Ottawa, ON: Health Canada; 2020.

8. National Institute on Aging. Closing the gaps: advancing emergency preparedness, response and recovery for older adults. Toronto, ON: NIA/Ryerson University; 2020. Available from: https://www.nia-ryerson.ca/commentary-posts/2020/12/14/ closing-the-gaps-advancing-emergency-preparedness-response -and-recovery-for-older-adults

9. International Longevity Centre Canada. Protecting human rights during and after COVID-19: challenges to the human rights of older people in Canada. The International Longevity Centre (ILC) Canada: Report to the United Nations Human Rights Commission. Ottawa, ON: University of Ottawa; 2020. Available from: https:// static1.squarespace.com/static/610bfbe35920f747f490fc $40 / \mathrm{t} / 6$ 11d366c4f53875c62138533/1629304428296/Final_Independent_Expert_on_Aging_-_report_on_the_Pandemic_in_Canada_202006_10_FINAL-converted.pdf

10. United Nations. Policy brief: The impact of COVID-19 on older persons. New York, NY: United Nations Report; 2020. Available from: https://unsdg.un.org/sites/default/files/2020-05/PolicyBrief-The-Impact-of-COVID-19-on-Older-Persons.pdf

11. Seitz D, Purandare N, Conn D. Prevalence of psychiatric disorders among older adults in long-term care homes: a systematic review. Int Psychogeratric. 2010;22(7):1025.

12. World Health Organization. Mental health of older adults: fact Sheet. Geneva, Switzerland: World Health Organization Media Centre, 2017. Available from: https://www.who.int/news-room/ fact-sheets/detail/mental-health-of-older-adults

13. Flint AJ, Bingham KS, Iaboni A. Effect of COVID-19 on the mental health care of older people in Canada. Int Psychogeriatr. 2020;32(10):1113-16.

14. Perlman C, Kirkham J, Velkers C, et al. Access to psychiatrist services for older adults in long-term care: A population-based study. J Am Med Direc Assoc. 2019;20(5):610-16.

15. Kuruvilla S, Sadana R, Montesinos EV, et al. A life-course approach to health: synergy with sustainable development goals. Bull WHO. 2018;96(1):42.

16. Graham N, Lindesay J, Katona C, et al. Reducing stigma and discrimination against older people with mental disorders: a technical consensus statement. Int J Geriatr Psychiatry. 2003; 18(8):670-78.

17. Holm AL, Lyberg A, Severinsson E. Living with stigma: depressed elderly persons' experiences of physical health problems. Nurs Res Pract. 2014;2014:527920.

18. Monahan C, Macdonald J, Lytle A, et al. COVID-19 and ageism: how positive and negative responses impact older adults and society. Am Psychol. 2020;75(7):887-96.

19. The Quebec Ombudsman. The Quebec Ombudsman's Status Report. COVID-19 in CHSLDs during the first wave of the pandemic. Learning from the crisis and moving to uphold the rights and dignity of CHSLD residents. Quebec City: Government of Quebec; 2020. Available from: https://protecteurducitoyen.qc.ca/ sites/default/files/pdf/rapports_speciaux/progress-report-chsldsCOVID-19.pdf

20. Picard A. Human rights don't have a best-before date: COVID-19 lays bare rampant ageism [online]. Globe and Mail. 2020 April 13.

21. Alkenbrack K. Doctors concerned about rise in dangerous medications in long-term care homes during pandemic [online]. CP24 News. 2020 December 3.

22. Wiseman SM, Crump RT, Sutherland JM. Surgical wait list management in Canada during a pandemic: many challenges ahead. Can J Surg. 2020;63(3):E226-28.

23. Thomas RK, Suleman R, Mackay M, et al. . Adapting to the impact of COVID-19 on mental health: an international perspective. J Psychiatry Neurosci. 2020;45(4):229-33.

24. Rosenberg S, Mendoza J, Tabatabaei-Jafari H, et al. International experiences of the active period of COVID-19-Mental health care. Health Policy Technol. 2020;9(4):503-09 
25. Joebges S, Biller-Andorno N. Ethics guidelines on COVID-19 triage - an emerging international consensus. Crit Care. 2020: 24(1):201.

26. Solomon MZ, Wynia MK, Gostin LO, et al. COVID-19 crisis triage — optimizing health outcomes and disability rights. $N$ Eng J Med. 2020;383(5):e27.

27. Holman N, Knighton P, Kar P, et al. Risk factors for COVID19-related mortality in people with type 1 and type 2 diabetes in England: a population-based cohort study. Lancet Diabetes Endocrinol. 2020;8(10):823-33.

28. Brown EE, Kumar S, Rajji TK, et al. Anticipating and Mitigating the Impact of COVID-19 Pandemic on Alzheimer's Disease and Related Dementias. Am J Geriatr Psychiatry. 2020;28(7):712-21. Epub 2020 Apr 18.

29. Van Rensbergen G, Nawrot T. Medical conditions of nursing home admissions. BMC Geriatr. 2010;10(1):46.

30. Iaboni A, Cockburn A, Marcil M, et al. Achieving safe, effective and compassionate quarantine or isolation of older adults with dementia in nursing homes. Am J Geriatr Psychiatry. 2020;28(8): 835-38.

31. Seitz DP, Brisbin S, Herrmann N, et al. Efficacy and feasibility of nonpharmacological interventions for neuropsychiatric symptoms of dementia in long term care: a systematic review. $J$ Am Med Dir Assoc. 2012;13(6):503-06.

32. Tupper SM, Ward H, Parmar J. Family presence in long-term care during the COVID-19 pandemic: call to action for policy, practice, and research. Can Geriatr J. 2020;23(4):335-339.

33. Seifert A, Batsis JA, Smith AC. Telemedicine in long-term care facilities during and beyond COVID-19: challenges caused by the digital divide. Front Public Health. 2020;8:601595.

34. World Health Organization. Strengthening the health system response to COVID-19. Policy Brief-Recommendations for the WHO European Region. Copenhagen, Denmark: WHO Regional Office for Europe; 2020. Available from: https://apps.who.int/ iris/handle/10665/333072

35. Centres for Disease Control and Prevention. Interim infection prevention and control recommendations for patients with suspected or confirmed Coronavirus disease 2019 (COVID-19) in healthcare settings. Atlanta, GA: CDCP; 2020. Available from: https://www.simlaweb.it/wp-content/uploads/2020/03/InfectionControl-Severe-acute-respiratory-syndrome-coronavirus2-SARS-CoV-2-CDC.pdf

36. Seifert A, Cotten SR, Xie B. A double burden of exclusion? Digital and social exclusion of older adults in times of COVID-19. J Gerontol B Psychol Sci Soc Sci. 2021;76(3):e99-3103.

37. Regional Geriatric Programs of Ontario. Listening to older adults about long term care on the International Day of Older Persons. 2020. Available from: https://rgps.on.ca/news/listening-to-olderadults-about-long-term-care-on-the-international-day-of-olderpersons/

38. Verbeek H, Gerritsen DL, Backhaus R, et al. Allowing visitors back in the nursing home during the COVID-19 crisis: a Dutch national study into first experiences and impact on well-being. J Am Med Dir Assoc. 2020;21(7):900-04.

39. Stall NM, Johnstone J, McGeer AJ, et al. Finding the right balance: an evidence-informed guidance document to support the re-opening of Canadian nursing homes to family caregivers and visitors during the Coronavirus disease 2019 pandemic. $J \mathrm{Am}$ Med Dir Assoc. 2020;21(10):1365-70.

40. Statistics Canada. Senior Care: difference by type of housing. No. 75-006-X. Ottawa, ON: Statistics Canada; 2015. https://www150. statcan.gc.ca/n1/pub/75-006-X/2015001/article/14142-eng.htm
41. Borrie M, Cooper T, Basu M, et al. Ontario geriatric specialist physician resources 2018. Can Geriatr J. 2020;23(3):219-27.

42. Cagnin A, Di Lorenzo R, Marra C, et al. Behavioral and psychological effects of coronavirus disease-19 quarantine in patients with dementia. Front Psychiatry. 2020;11:916.

43. Shea YF, Shum CK, Wan WH, et al. Worsening behavioural and psychological symptoms of dementia during the coronavirus disease 2019 pandemic. Psychogeriatrics. 2020;20(6):916-17.

44. Canadian Coalition for Seniors Mental Health. The assessment and treatment of mental health issues in long-term care homes: (Focus on mood and behavior symptoms). 2014 Guideline Update. Toronto, ON: The Coalition; 2014. Available from: https:// ccsmh.ca/wp-content/uploads/2016/03/2014-ccsmh-GuidelineUpdate-LTC.pdf

45. MacCourt P, Wilson K, Tourigny-Rivard MF. Guidelines for comprehensive mental health services for older adults in Canada. Calgary, AB: Mental Health Commission of Canada; 2011. Available from: https://www.mentalhealthcommission.ca/sites/ default/files/2017-09/mhcc_seniors_guidelines_0.pdf

46. Mental Health First Aid Canada. Seniors [online course]. Ottawa, ON: MHFA; 2020. Available from: https://www.mhfa.ca/ en/course-type/seniors

47. Soares WB, Silvestre IT, Lima AM, et al. The influence of telemedicine care on the management of behavioral and psychological symptoms in dementia (BPSD) risk factors induced or exacerbated during the COVID-19 pandemic. Front Psychiatry. 2020;11:577629.

48. Waypoint Centre for Mental Health Care. Discussion Paper: The need for a multi-dimensional strategy to address the care of older adults \& their caregivers in COVID-19. Penetanguishene, ON: Waypoint Centre; 2020. Available from: https://www.nsmsgs. $\mathrm{ca} /$ Uploads/ContentDocuments/Multi\%20Dimensional\%20 Strategy\%20Discussion\%20Paper\%20May13-20.pdf

49. McGilton KS, Escrig-Pinol A, Gordon A, et al. Uncovering the devaluation of nursing home staff during COVID-19: are we fuelling the next health care crisis? J Am Med Direc Assoc. 2020;21(7):962-65.

50. World Psychiatric Association. Psychiatry and the COVID-19 pandemic [position statement]. Geneva, Switzerland: WPA Secretariat; 2020. Available from: https://3ba346de-fde6-473fb1da-536498661f9c.filesusr.com/ugd/e172f3_43fb0fa815ef495 e9fdaaf331da6ff97.pdf

51. Bethell J, Aelick K, Babineau J, et al. Social connection in longterm care homes: A scoping review of published research on the mental health impacts and potential strategies during COVID- 19 . J Am Med Dir Assoc. 2021;22(2):228-37. Online ahead of print.

52. Burrows AB, Morris JN, Simon SE, et al. Development of a minimum data set-based depression rating scale for use in nursing homes. Age Aging. 2000;29(2):165-72.

53. Perlman CM, Hirdes JP. The aggressive behavior scale: a new scale to measure aggression based on the minimum data set. $\mathrm{J} \mathrm{Am}$ Geriatr Soc. 2008;56(12):2298-303.

54. Morris JN, Fries BE, Mehr DR, et al. MDS cognitive performance scaleC. J Gerontol. 1994;49(4):M174-M182.

Correspondence to: Dallas Seitz, MD, PhD, Department of Psychiatry, Cumming School of Medicine, Room 2919 HSC, University of Calgary, 3330 Hospital Dr. NW, Calgary, AB T2N 4N1

E-mail: dallas.seitz@ucalgary.ca 